\title{
Cloaking via anomalous localized resonance for doubly complementary media in the finite frequency regime
}

\author{
Hoai-Minh Nguyen * \\ January 1, 2015
}

\begin{abstract}
Cloaking a source via anomalous localized resonance (ALR) was discovered by Milton and Nicorovici in 14. A general setting in which cloaking a source via ALR takes place is the settting of doubly complementary media. This was introduced and studied in 19 for the quasistatic regime. In this paper, we study cloaking a source via ALR for doubly complementary media in the finite frequency regime as a natural continuation of [19]. We establish the following results: 1) Cloaking a source via ALR appears if and only if the power blows up; 2) The power blows up if the source is "placed" near the plasmonic structure; 3) The power remains bounded if the source is far away from the plasmonic structure. Concerning the analysis, we extend ideas from [19] and add new insights on the problem which allows us to overcome difficulties related to the finite frequency regime and to obtain new information on the problem. In particular, we are able to characterize the behaviour of the fields far enough from the plasmonic shell as the loss goes to 0 for an arbitrary source outside the core-shell structure in the doubly complementary media setting.
\end{abstract}

\section{Introduction}

Negative index materials (NIMs) were first investigated theoretically by Veselago in [26]. The existence of such materials was confirmed by Shelby, Smith, and Schultz in [25]. The study of NIMs has attracted a lot attention in the scientific community thanks to their many applications. One of the appealing ones is cloaking. There are at least three ways to do cloaking using NIMs. The first one is based on plasmonic structures introduced by Alu and Engheta in [3]. The second one uses the concept of complementary media. This was suggested by Lai et al. in [12] and confirmed theoretically in [20] for inspired schemes. The last one is based on the concept of ALR discovered by Milton and Nicorovici in [14]. In this paper, we concentrate on the last method.

Cloaking a source via ALR was discovered by Milton and Nicorovici in [14. Their work has root from [24] (see also [13]) where the localized resonance was observed and established for constant symmetric plasmonic structures in the two dimensional quasistatic regime. More

*EPFL SB MATHAA CAMA, Station 8, CH-1015 Lausanne, hoai-minh.nguyen@epfl.ch 
precisely, in [14], the authors studied core-shell plasmonic structures in which a circular shell has permittivity $-1-i \delta$ while its complement has permittivity 1 where $\delta$ denotes the loss of the material in the shell 1 . Let $r_{1}$ and $r_{2}$ be the inner and the outer radius of the shell. They showed that there is a critical radius $r_{*}:=\left(r_{2}^{3} r_{1}^{-1}\right)^{1 / 2}$ such that a dipole is not seen by an observer away from the core-shell structure, hence it is cloaked, if and only if the dipole is within distance $r_{*}$ of the shell. Moreover, the power $E_{\delta}\left(u_{\delta}\right)$ of the field $u_{\delta}$, which is defined in (1.5), blows up as the loss $\delta$ goes to 0 . Two key features of this phenomenon are: 1) the localized resonance, i.e., the fields blow up in some regions and remain bounded in some others as the loss goes to $0 ; 2$ ) the connection between the localized resonance and the blow up of the power as the loss goes to 0 .

Cloaking a source via ALR has been mainly studied in the quasistatic regime. In [7], Bouchitte and Schweizer proved that a small circular inclusion of radius $\gamma(\delta)$ (with $\gamma(\delta) \rightarrow 0$ fast enough) is cloaked by the core-shell plasmonic structure mentioned above in the two dimensional quasistatic regime if the inclusion is located within distance $r_{*}$ of the shell. Otherwise it is visible. Concerning the second feature of cloaking a source via ALR, the blow up of the power was studied for a more general setting in two dimensional quasistatic regime by Ammari et al. in [5] and Kohn et al. in [11. More precisely, they considered nonradial core-shell structures in which the shell has permittivity $-1-i \delta$ and its complement has permitivity 1. In [5], Ammari et al. dealt with arbitrary shells and provided a characterization of sources for which the power blows up via the information of the spectral decomposition of the Neumann-Poincaré type operator. In [11], Kohn et al. considered core-shell structures in which the outer boundary of the shell is round but the inner is not and established the blow up of the power for some class of sources using a variational approach. A connection between the blow up of the power and the localized resonance subtly depends on the geometry and property of plasmonic structures. It was showed in [22] that such a connection does not hold in general. Cloaking a source via ALR in some special three dimensional geometry was studied in [4. Motivated by the concept of reflecting complementary media suggested and studied in [16] and results mentioned above, in [19] we studied cloaking a source via ALR for a general core shell structure of doubly complementary media property (see Definition 1.2) in the quasistatic regime. $2^{2}$ More precisely, we established the following three properties for doubly complementary media:

P1) Cloaking a source via ALR appears if and only if the power blows up.

P2) The power blows up if the source is located "near" the shell.

P3) The power remains bounded if the source is far away from the shell.

Using these results, we extended various results mentioned previously. Moreover, we were able to obtain schemes to cloak an arbitrary source concentrating on an arbitrary smooth bounded manifold of codimension 1 placed in an arbitrary medium via ALR; the cloak is independent

\footnotetext{
${ }^{1}$ In fact, in [14] and in other works, the authors consider the permittivity $-1+i \delta$ instead of $-1-i \delta$; but this point is not essential.

${ }^{2}$ Roughly speaking, the plasmonic shell is not only complementary with a part of the complement of the core shell but also complement to a part of the core.
} 
of the source. The analysis in [19] is on one hand based on the reflecting techniques initiated in [16], the removing localized technique introduced in [17, 20] to deal with the localized resonance. On the other hand, the analysis in [19] is based on new observations on the Cauchy problems and the separation of variables technique for a general shell introduced there. The implement of this technique is an ad-hoc part of [19].

In this paper, we study cloaking a source via ALR for the finite frequency regime as a natural continuation of [19]. More precisely, we establish Properties P1), P2) and P3) for doubly complementary media in the finite frequency regime. As a consequence, we are also able, as in the quasistatic regime, to obtain schemes to cloak a non generic arbitrary source concentrating on an arbitrary smooth bounded manifold of codimension 1 placed in an arbitrary medium via ALR; the cloak is independent of the source (see Section 6). Concerning the analysis, we extend ideas from [19] and add various new insights for the problem which allows us to overcome difficulties related to the finite frequency regime such as the use of the maximum priniciple, to shorten the approach in [19], and to obtain new information on the cloaking a source via ALR. In particular, we can characterize the behaviour of the fields far enough from the plasmonic shell as the loss goes to 0 for arbitrary sources in the doubly complementary media setting (Theorem 1.1). This fact is interesting in itself and new to our knowledge.

Let $k>0$ and let $A$ be a (real) uniformly elliptic symmetric matrix defined in $\mathbb{R}^{d}(d \geq 2)$, and $\Sigma$ be a bounded real function defined in $\mathbb{R}^{d}$. Assume that

$$
A(x)=I \quad \Sigma(x)=1 \text { for }|x| \text { large enough }
$$

and

$$
A \text { is piecewise } C^{1} \text {. }
$$

Let $\Omega_{1} \subset \subset \Omega_{2} \subset \subset \mathbb{R}^{d}$ be smooth connected open subsets of $\mathbb{R}^{d}$, and set, for $\delta \geq 0$,

$$
s_{\delta}(x)=\left\{\begin{array}{cl}
-1-i \delta & \text { in } \Omega_{2} \backslash \Omega_{1}, \\
1 & \text { in } \mathbb{R}^{d} \backslash\left(\Omega_{2} \backslash \Omega_{1}\right) .
\end{array}\right.
$$

For $f \in L_{\mathrm{c}}^{2}\left(\mathbb{R}^{d}\right)$ with $\operatorname{supp} f \cap B_{r_{2}}=\varnothing$ and $\delta>0$, let $u_{\delta} \in H_{\text {loc }}^{1}(\Omega)$ be the unique outgoing solution to

$$
\operatorname{div}\left(s_{\delta} A \nabla u_{\delta}\right)+k^{2} s_{0} \Sigma u_{\delta}=f \text { in } \mathbb{R}^{d} .
$$

Here and in what follows

$$
L_{\mathrm{c}}^{2}\left(\mathbb{R}^{d}\right):=\left\{f \in L^{2}\left(\mathbb{R}^{d}\right) \text { with compact support }\right\} .
$$

For $R>0$ and $x \in \mathbb{R}^{d}$, we also denote $B(x, R)$ the ball in $\mathbb{R}^{d}$ centered at $x$ and of radius $R$; when $x=0$, we simply denote $B(x, R)$ by $B_{R}$. Recall that a function $u \in H_{l o c}^{1}\left(\mathbb{R}^{d} \backslash B_{R}\right)$ for some $R>0$ which is a solution to the equation $\Delta u+k^{2} u=0$ in $\mathbb{R}^{d} \backslash B_{R}$ is said to satisfy the outgoing condition if

$$
\partial_{r} u-i k u=o\left(r^{\frac{1-d}{2}}\right) \text { as } r=|x| \rightarrow+\infty .
$$


The power $E_{\delta}\left(u_{\delta}\right)$ is defined by (see, e.g., [14])

$$
E_{\delta}\left(u_{\delta}\right)=\delta \int_{\Omega_{2} \backslash \Omega_{1}}\left|\nabla u_{\delta}\right|^{2}
$$

The normalization of $u_{\delta}$ is $v_{\delta} \in H_{l o c}^{1}\left(\mathbb{R}^{d}\right)$ which is the unique outgoing solution of

$$
\operatorname{div}\left(s_{\delta} A \nabla v_{\delta}\right)+k^{2} s_{0} \Sigma v_{\delta}=f_{\delta} \text { in } \mathbb{R}^{d} .
$$

Here

$$
f_{\delta}=c_{\delta} f
$$

and $c_{\delta}$ is the normalization constant such that

$$
\delta^{1 / 2} \int_{B_{r_{2}} \backslash B_{r_{1}}}\left|\nabla v_{\delta}\right|^{2}=1 .
$$

In this paper, we establish properties $\mathrm{P} 1), \mathrm{P} 2)$, and $\mathrm{P} 3)$ for $(A, \Sigma)$ of doubly complementary property. Before giving the definition of doubly complementary media for a general core-shell structure, let us recall the definition of reflecting complementary media introduced in [16, Definition 1].

Definition 1.1 (Reflecting complementary media). Let $\Omega_{1} \subset \subset \Omega_{2} \subset \subset \Omega_{3} \subset \subset \mathbb{R}^{d}$ be smooth open bounded subsets of $\mathbb{R}^{d}$. The media $(A, \Sigma)$ in $\Omega_{3} \backslash \Omega_{2}$ and $(-A,-\Sigma)$ in $\Omega_{2} \backslash \Omega_{1}$ are said to be reflecting complementary if there exists a diffeomorphism $F: \Omega_{2} \backslash \bar{\Omega}_{1} \rightarrow \Omega_{3} \backslash \bar{\Omega}_{2}$ such that

$$
\begin{gathered}
\left(F_{*} A, F_{*} \Sigma\right)=(A, \Sigma) \text { for } x \in \Omega_{3} \backslash \Omega_{2}, \\
F(x)=x \text { on } \partial \Omega_{2},
\end{gathered}
$$

and the following two conditions hold: 1) There exists an diffeomorphism extension of $F$, which is still denoted by $F$, from $\Omega_{2} \backslash\left\{x_{1}\right\} \rightarrow \mathbb{R}^{d} \backslash \bar{\Omega}_{2}$ for some $\left.x_{1} \in \Omega_{1} ; 2\right)$ There exists a diffeomorphism $G: \mathbb{R}^{d} \backslash \bar{\Omega}_{3} \rightarrow \Omega_{3} \backslash\left\{x_{1}\right\}$ such that

$$
G(x)=x \text { on } \partial \Omega_{3},
$$

and

$$
G \circ F: \Omega_{1} \rightarrow \Omega_{3} \text { is a diffeomorphism if one sets } G \circ F\left(x_{1}\right)=x_{1} .
$$

Here and in what follows, if $T$ is a diffeomorphism and $a$ and $\sigma$ are a matrix-valued function and a complex function, we use the following standard notations

$$
T_{*} a(y)=\frac{D T(x) a(x) D T(x)^{T}}{|\operatorname{det} D T(x)|} \quad \text { and } \quad T_{*} \sigma(y)=\frac{\sigma(x)}{|\operatorname{det} D T(x)|} \quad \text { where } x=T^{-1}(y) .
$$

In (1.9) and (1.10), $F$ and $G$ denote some diffeomorphism extensions of $F$ and $G$ in a neighborhood of $\partial \Omega_{2}$ and of $\partial \Omega_{3}$. Conditions $(1.8)$ and $(1.9)$ are the main assumptions in Definition 1.1. The key point behind this requirement is roughly speaking the following property: if $u_{0} \in H^{1}\left(\Omega_{3} \backslash \Omega_{1}\right)$ is a solution of $\operatorname{div}\left(s_{0} A \nabla u_{0}\right)+k^{2} s_{0} \Sigma u_{0}=0$ in $\Omega_{3} \backslash \Omega_{2}$ 
and if $u_{1}$ defined in $\Omega_{3} \backslash \Omega_{2}$ by $u_{1}=u_{0} \circ F^{-1}$, then $\operatorname{div}\left(A \nabla u_{1}\right)+k^{2} \Sigma u_{1}=0$ in $\Omega_{3} \backslash \Omega_{2}$, $u_{1}-u_{0}=A \nabla\left(u_{1}-u_{0}\right) \cdot \nu=0$ on $\partial \Omega_{2}$ (see [16, Lemma 2]). Hence $u_{1}=u$ in $\Omega_{3} \backslash \Omega_{2}$ by the unique continuation principle. Conditions 1) and 2) are mild assumptions. Introducing $G$ makes the analysis more accessible, see [16, 17, 20, 23] and the analysis presented in this paper.

Remark 1.1. The class of reflecting complementary media has played an important role in the other applications of NIMs such as cloaking and superlensing using complementary see [17, 20, 23].

Remark 1.2. Let $d=2, A=I, 0<r_{1}<r_{2}<+\infty$ and set $r_{3}=r_{2}^{2} / r_{1}$. Letting $F$ be the Kelvin transform with respect to $\partial B_{r_{2}}$, i.e., $F(x)=r_{2}^{2} x /|x|^{2}$ and $\Omega_{i}=B_{r_{i}}$, one can verify that in the quasistatic regime the core-shell structures considered by Milton and Nicorovici in [14] and by Kohn et al. in [11] have the reflecting complementary property.

We are ready to introduce the concept of doubly complementary media for the finite frequency regime.

Definition 1.2. The medium $\left(s_{0} A, s_{0} \Sigma\right)$ is said to be doubly complementary if for some $\Omega_{2} \subset \subset \Omega_{3},(A, \Sigma)$ in $\Omega_{3} \backslash \Omega_{2}$ and $(-A,-\Sigma)$ in $\Omega_{2} \backslash \Omega_{1}$ are reflecting complementary, and

$$
F_{*} A=G_{*} F_{*} A=A \quad \text { and } \quad F_{*} \Sigma=G_{*} F_{*} \Sigma=\Sigma \text { in } B_{r_{3}} \backslash B_{r_{2}},
$$

for some $F$ and $G$ coming from Definition 1.1 (see Figure 1).

The reason for which media satisfying (1.13) are called doubly complementary media is that $(-A,-\Sigma)$ in $B_{r_{2}} \backslash B_{r_{1}}$ is not only complementary to $(A, \Sigma)$ in $\Omega_{3} \backslash \Omega_{2}$ but also to $(A, \Sigma)$ in $(G \circ F)^{-1}\left(\Omega_{3} \backslash \overline{\Omega_{2}}\right)$ (a subset of $\Omega_{1}$ ) (see [18]). The key property behind Definition 1.2 is as follows. Assume $u_{0} \in H_{l o c}^{1}\left(\mathbb{R}^{d}\right)$ is a solution of (1.4) with $\delta=0$ and set $u_{1}=u \circ F^{-1}$ and $u_{2}=u_{1} \circ G^{-1}$. Then $u_{1}, u_{2}$ satisfy the equation $\operatorname{div}(A \nabla \cdot)+k^{2} \Sigma \cdot=0$ in $\Omega_{3} \backslash \Omega_{2}$ (see [16, Lemma 2]).

Remark 1.3. Taking $d=2, A=I$ and $r_{3}=r_{2}^{2} / r_{1}$, and letting $F$ and $G$ be the Kelvin transform with respect to $\partial B_{r_{2}}$ and $\partial B_{r_{3}}$, one can verify that the core-shell structures considered by Milton and Nicorovici in [14] is of doubly complementary property. It is worthy to note that one requires no information of $A$ outside $B_{r_{3}}$ and inside $B_{r_{1}^{2} / r_{2}}$ in the definition of doubly complementary media.

Remark 1.4. Given $(A, \Sigma)$ in $\mathbb{R}^{d}$ it is not easy in general to verify whether or not $\left(s_{0} A, s_{0} \Sigma\right)$ is doubly complementary. Nevertheless, given $\Omega_{1} \subset \Omega_{2} \subset \subset \Omega$ and $(A, \Sigma)$ in $\Omega_{3} \backslash \Omega_{2}$, it is quite easy to choose $(A, \Sigma)$ in $\Omega_{2}$ such that $\left(s_{0} A, s_{0} \Sigma\right)$ is doubly complementary. One just needs to choose diffeomorphisms $F$ and $G$ as in Definition 1.1 and define $(A, \Sigma)=\left(F_{*}^{-1} A, F_{*}^{-1} \Sigma\right)$ in $\Omega_{2} \backslash \Omega_{1}$ and $(A, \Sigma)=\left(G_{*}^{-1} F_{*}^{-1} A, G^{-1} * F_{*}^{-1} \Sigma\right)$ in $G^{-1} \circ F^{-1}\left(\Omega_{3} \backslash \Omega_{2}\right)$. This idea is used in Section 6 when we discuss cloaking sources in an arbitrary medium.

The main result of this paper is the following theorem which reveals the behavior of $u_{\delta}$ for a general source $f$. 


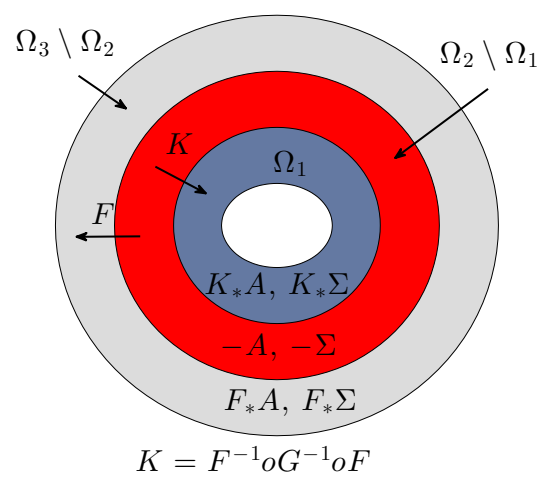

Figure 1: $\left(s_{0} A, s_{0} \Sigma\right)$ is doubly complementary: $(-A,-\Sigma)$ in $\Omega_{2} \backslash \Omega_{1}$ (the red region) is complementary to $\left(F_{*} A, F_{*} \Sigma\right)$ in $\Omega_{3} \backslash \Omega_{2}$ (the grey region) and $\left(K_{*} A, K_{*} \Sigma\right)$ with $K=F^{-1} \circ$ $G^{-1} \circ F$ in $K\left(B_{r_{2}} \backslash B_{r_{1}}\right)$ (the blue grey region).

Theorem 1.1. Let $d \geq 2, k>0, f \in L_{\mathrm{c}}^{2}\left(\mathbb{R}^{d}\right)$ with $\operatorname{supp} f \cap \Omega_{2}=\varnothing$, and let $u_{\delta} \in H_{\text {loc }}^{1}\left(\mathbb{R}^{d}\right)$ be the unique outgoing solution of (1.4). Assume that $\left(s_{0} A, s_{0} \Sigma\right)$ is doubly complementary. Then

$$
u_{\delta} \rightarrow \hat{u} \text { weakly in } H_{l o c}^{1}\left(\mathbb{R}^{d} \backslash B_{r_{3}}\right) \text {, }
$$

where $\hat{u} \in H_{\text {loc }}^{1}\left(\mathbb{R}^{d}\right)$ is the unique outgoing solution of

$$
\operatorname{div}(\hat{A} \nabla \hat{u})+k^{2} \hat{\Sigma} \hat{u}=f \text { in } \mathbb{R}^{d} .
$$

Here

$$
(\hat{A}, \hat{\Sigma}):=\left\{\begin{array}{cl}
(A, \Sigma) & \text { in } \mathbb{R}^{d} \backslash \Omega_{3}, \\
\left(G_{*} F_{*} A, G_{*} F_{*} \Sigma\right) & \text { in } \Omega_{3} .
\end{array}\right.
$$

Using Theorem 1.1, one can establish the equivalence between the blow up of the power and the cloaking via ALR as follows. Suppose that the power blows up, i.e.,

$$
\lim _{n \rightarrow \infty} \delta_{n}\left\|\nabla u_{\delta_{n}}\right\|_{L^{2}\left(B_{r_{2}} \backslash B_{r_{1}}\right)}^{2}=+\infty .
$$

Then, by Theorem 1.1, $v_{\delta_{n}} \rightarrow 0$ in $\mathbb{R}^{d} \backslash B_{r_{3}}$; the localized resonance takes place. The source $\alpha_{\delta_{n}} f$ is not seen by observers far away from the shell: the source is cloaked. If the power $E_{\delta_{n}}\left(u_{\delta_{n}}\right)$ remains bounded, then $u_{\delta_{n}} \rightarrow \hat{u}$ weakly in $H_{\text {loc }}^{1}\left(\mathbb{R}^{d} \backslash B_{r_{3}}\right)$. Since $\hat{u} \in H_{\text {loc }}^{1}\left(\mathbb{R}^{d}\right)$ is the unique outgoing solution to (1.15, the source is not cloaked.

Remark 1.5. It follows from (1.12) that if $\left(s_{0} A, s_{0} \Sigma\right)$ is doubly complementary media then $(A, \Sigma)$ is not piecewise constant. This is the reason for which there are very few results on cloaking via ALR in the finite frequency regime in both two and three dimensions.

In comparison with results in [19, Theorem 1.1 is stronger: no conditions on the blow up rate of the power is required. The proof of Theorem 1.1 is in the spirit of [19]. Nevertheless, 
we incorporate two important ingredients. The first one is on the blow up rate of the power of $u_{\delta}$ in (2.15) which is derived in this paper but was assumed previously. The second one is on the removing localized singularity technique. Here, we are able to construct in a simple and robust way the singular part which is necessary to removed. This helps us to avoid the ad-hoc separation of variables for a general shell developed and implemented in [19]. The construction of the removing term comes from a remark of Etienne Sandier. The author would like to thank him for it. To our knowledge, Theorem 1.1 is new and is the first result providing the connection between the blow up of the power and the invisibility of a source in the finite frequency regime.

Concerning the blow up of the power, we can prove the following result which holds for a large class of media in which the reflecting complementary property holds only locally.

Proposition 1.1. Assume that there exists a diffeomorphism $F: \Omega_{2} \backslash \Omega_{1} \rightarrow \Omega_{3} \backslash \Omega_{2}$ for some $\Omega_{2} \subset \subset \Omega_{3} \subset \mathbb{R}^{d}$ such that $F(x)=x$ on $\partial \Omega_{2}$,

$$
(A, \Sigma)=\left(F_{*} A, F_{*} \Sigma\right) \text { in } D \text { where } D:=B\left(x_{0}, R_{0}\right) \cap\left(\Omega_{3} \backslash \Omega_{2}\right) .
$$

for some $x_{0} \in \partial \Omega_{2}$ and $R_{0}>0$. Let $f \in L_{\mathrm{c}}^{2}\left(\mathbb{R}^{d}\right)$ and assume that $A$ is Lipschitz in $\bar{D}$. There exists $0<r_{0}<R_{0}$, independent of $f$, such that if there is no solution $v \in H^{1}\left(D_{1}\right)$ where $D_{1}:=D \cap B\left(x_{0}, r_{0}\right)$ to the Cauchy problem:

$$
\operatorname{div}(A \nabla v)+k^{2} \Sigma v=f \text { in } D_{1} \quad \text { and } \quad v=A \nabla v \cdot \nu=0 \text { on } \partial D_{1} \backslash \partial B\left(x_{0}, r_{0}\right),
$$

then

$$
\limsup _{\delta \rightarrow 0} \delta \int_{\Omega_{2} \backslash \Omega_{1}}\left|\nabla u_{\delta}\right|^{2}=+\infty
$$

where $u_{\delta} \in H_{\text {loc }}^{1}\left(\mathbb{R}^{d}\right)$ is the unique outgoing solution of 1.4 .

The proof of Proposition 1.1 essentially uses the ideas in the one of [21, Lemma 10] which has root from [19]. It is clear that Proposition 1.1 implies Property P2). Note that $\left(s_{0} A, s_{0} \Sigma\right)$ is not required to be doubly complementary in Proposition 1.1.

Concerning the boundedness of the power, we have the following result, which implies Property P3).

Proposition 1.2. Let $d=2,3,0<\delta<1, f \in L_{\mathrm{c}}^{2}\left(\mathbb{R}^{d}\right)$, and let $u_{\delta} \in H^{1}\left(\mathbb{R}^{d}\right)$ be the unique solution (1.4). Assume that $\left(s_{0} A, s_{0} \Sigma\right)$ is doubly complementary media. We have, if supp $f \cap \Omega_{3}=\varnothing$ then

$$
\left\|u_{\delta}\right\|_{H^{1}\left(B_{R}\right)} \leq C_{R}\|f\|_{L^{2}}
$$

for some $C_{R}$ independent of $f$ and $\delta$.

When $\Omega_{j}=B_{r_{j}}$ for $j=2,3$ and $A=I$ in $\Omega_{3} \backslash \Omega_{2}$, more quantitative estimates on the blow up and the boundedness of the power are given in the following proposition in the spirit of [19, Theorems 1.2 and 1.3] (inspired by [5, 11]).

Proposition 1.3. Let $d=2,3, f \in L_{\mathrm{c}}^{2}\left(\mathbb{R}^{d}\right)$, and let $u_{\delta} \in H^{1}\left(\mathbb{R}^{d}\right)$ be the unique solution of (1.4). Assume that $\left(s_{0} A, s_{0} \Sigma\right)$ is doubly complementary media, $\Omega_{2}=B_{r_{2}}$ and $\Omega_{3}=B_{r_{3}}$ for some $0<r_{2}<r_{3}$, and $(A, \Sigma)=(I, 1)$ in $B_{r_{3}} \backslash B_{r_{2}}$. We have 
1. If there exists $w \in H^{1}\left(B_{r_{0}} \backslash B_{r_{2}}\right)$ for some $r_{0}>\sqrt{r_{2} r_{3}}$ with the properties

$$
\operatorname{div}(A \nabla w)+k^{2} \Sigma w=f \text { in } B_{r_{0}} \backslash B_{r_{2}} \quad \text { and } \quad w=A \nabla w \cdot \nu=0 \text { on } \partial B_{r_{2}},
$$

then

$$
\limsup _{\delta \rightarrow 0} \delta\left\|u_{\delta}\right\|_{H^{1}\left(B_{r_{3}}\right)}^{2}<+\infty .
$$

2. If there does not exist $v \in H^{1}\left(B_{r_{0}} \backslash B_{r_{2}}\right)$ for some $r_{0}<\sqrt{r_{2} r_{3}}$ with the properties

$$
\operatorname{div}(A \nabla v)+k^{2} \Sigma v=f \text { in } B_{r_{0}} \backslash B_{r_{2}} \quad \text { and } \quad v=A \nabla v \cdot \nu=0 \text { on } \partial B_{r_{2}},
$$

then

$$
\liminf _{\delta \rightarrow 0} \delta\left\|\nabla u_{\delta}\right\|_{L^{2}\left(B_{r_{3}} \backslash B_{r_{2}}\right)}^{2}=+\infty .
$$

One only assumes that $(A, \Sigma)=(I, 1)$ in $B_{r_{3}} \backslash B_{r_{2}}$ : the separation of variables is out of reach here. The proof of the first statement of Proposition 1.3 is based on a kind of removing singularity technique and has roots from [19]. A key point is the construction of the auxiliary function $W_{\delta}$ in (5.8). The proof of the second statement is based on an observation on a Cauchy problem in [19] and involves a three spheres inequality.

We finally point out that the stability of the Helmholtz with sign changing coefficients was studied in [1, 9, 10] and references therein by the integral method, the pseudo differential operator theory, and the T-coercivity approach and was unified and generalized in [21] using different techniques based on reflections and the study of Cauchy's problems. It was also showed in 21] that complementary property is necessary for the appearance of resonance.

The paper is organized as follows. The proof of Theorem 1.1 is given in Section 2 . Sections 3, 4, and 5 are devoted to the proofs of Propositions 1.1, 1.2, and 1.3 respectively. Finally, in Section 6, we present schemes of cloaking a general class of sources via ALR in arbitrary medium for the finite frequency regime.

\section{Proof of Theorem 1.1}

We start this section with a lemma on the stability of 1.4 which is used repeatedly in this paper.

Lemma 2.1. Let $d=2,3, R_{0}>0, k>0, \delta \in(0,1), R_{0}>0, g \in H^{-1}\left(\mathbb{R}^{d}\right)$ with support in $B_{R_{0}}$. Then there exists a unique outgoing solution $v_{\delta} \in H_{\text {loc }}^{1}\left(\mathbb{R}^{d}\right)$ to the equation

$$
\operatorname{div}\left(s_{\delta} A \nabla v_{\delta}\right)+k^{2} s_{0} \Sigma v_{\delta}=g \text { in } \mathbb{R}^{d} .
$$

Moreover,

$$
\left\|v_{\delta}\right\|_{H^{1}\left(B_{R}\right)}^{2} \leq \frac{C_{R}}{\delta}\left|\int g \bar{v}_{\delta}\right|
$$

for some positive constant $C_{R}$ independent of $g$ and $\delta$, as $\delta$ is small. 
Proof. The existence of $v_{\delta}$ follows from the uniqueness of $v_{\delta}$ by using the limiting absorption principle. We now establish the uniqueness of $v_{\delta}$ by showing that $v_{\delta}=0$ if $v_{\delta} \in H_{l o c}^{1}\left(\mathbb{R}^{d}\right)$ is an outgoing solution of

$$
\operatorname{div}\left(s_{\delta} A \nabla v_{\delta}\right)+k^{2} s_{0} \Sigma v_{\delta}=0 \text { in } \mathbb{R}^{d} .
$$

Multiplying the above equation by $\bar{v}_{\delta}$ (the conjugate of $v_{\delta}$ ) and integrating the obtained expression on $B_{R}$ with $R \geq R_{0}$, we have

$$
\int_{B_{R}} s_{\delta}\left\langle A \nabla v_{\delta}, \nabla v_{\delta}\right\rangle d x-\int_{B_{R}} k^{2} s_{0} \Sigma\left|v_{\delta}\right|^{2} d x-\int_{\partial B_{R}} \partial_{r} v_{\delta} \bar{v}_{\delta}=0 .
$$

By letting $R \rightarrow+\infty$, considering the imaginary part, and using the outgoing condition, we obtain

$$
\int_{\Omega_{2} \backslash \Omega_{1}}\left\langle A \nabla v_{\delta}, \nabla v_{\delta}\right\rangle d x=0
$$

Since $A$ is uniformly elliptic, it follows that $v_{\delta}$ is constant in $\Omega_{2} \backslash \Omega_{1}$. Thus $v_{\delta}=0$ in $\Omega_{2} \backslash \Omega_{1}$ since $\operatorname{div}\left(s_{\delta} A \nabla v_{\delta}\right)+k^{2} s_{0} \Sigma v_{\delta}=0$ in $\Omega_{2} \backslash \Omega_{1}$. This implies $v_{\delta}=0$ in $\mathbb{R}^{d} \backslash \Omega_{2}$ and $v_{\delta}=0$ in $\Omega_{1}$ by the unique continuation principle. The proof for the uniqueness of $v_{\delta}$ is complete.

Without loss of generality, one may assume that such that (1.1) holds for $|x| \geq R_{0}$ and $\Omega_{2} \subset \subset B_{R_{0}}$. We next establish $(2.2)$ with $R=R_{0}$ by contradiction. Assume that $(2.2)$ is not true. Then there exists $\left(g_{\delta}\right) \subset H^{-1}\left(\mathbb{R}^{d}\right)$ such that

$$
\left\|v_{\delta}\right\|_{H^{1}\left(B_{R_{0}}\right)}=1 \text { and } \frac{1}{\delta}\left|\int g_{\delta} \bar{v}_{\delta}\right| \rightarrow 0,
$$

as $\delta \rightarrow 0$, where $v_{\delta} \in H_{\text {loc }}^{1}\left(\mathbb{R}^{d}\right)$ is the unique solution to the equation

$$
\operatorname{div}\left(s_{\delta} A \nabla v_{\delta}\right)+k^{2} s_{0} \Sigma v_{\delta}=g_{\delta} \text { in } \mathbb{R}^{d} .
$$

In fact, by contradiction these properties only hold for a sequence of $\left(\delta_{n}\right) \rightarrow 0$. However, for simplicity of the notation, we still use $\delta$ instead of $\delta_{n}$ to denote an element of such a sequence. Since (see e.g., [15, Lemma 2.3])

$$
\left\|v_{\delta}\right\|_{H^{1}\left(B_{R} \backslash B_{R_{0}}\right)} \leq C_{R}\left\|v_{\delta}\right\|_{H^{1 / 2}\left(\partial B_{R_{0}}\right)},
$$

without loss of generality, one may assume that $v_{\delta} \rightarrow v_{0}$ in $L_{l o c}^{2}\left(\mathbb{R}^{d}\right)$ and weakly in $H_{l o c}^{1}\left(\mathbb{R}^{d}\right)$ for some $v_{0} \in H_{l o c}^{1}\left(\mathbb{R}^{d}\right)$. Multiplying (2.4) by $\bar{v}_{\delta}$ and integrating the obtained expression in $B_{R}$ with $R \geq R_{0}$, we have

$$
\int_{B_{R}} s_{\delta}\left\langle A \nabla v_{\delta}, \nabla v_{\delta}\right\rangle d x-\int_{B_{R}} k^{2} s_{0} \Sigma\left|v_{\delta}\right|^{2} d x=-\int_{B_{R}} g_{\delta} \bar{v}_{\delta} d x+\int_{\partial B_{R}} \partial_{r} v_{\delta} \bar{v}_{\delta} .
$$

Letting $\delta \rightarrow 0$, by 2.3 , we obtain

$$
\Im\left(\int_{\partial B_{R}} \partial_{r} v_{0} \bar{v}_{0}\right)=0
$$


Using the fact that $v_{0}$ satisfies the outgoing condition, by letting $R \rightarrow+\infty$ and considering the imaginary part in (2.7), we obtain

$$
\int_{\partial B_{R}}\left|v_{0}\right|^{2} \rightarrow 0 \text { as } \delta \rightarrow 0
$$

It follows from Reillich's lemma that $v_{0}=0$ in $\mathbb{R}^{d} \backslash B_{R_{0}}$. One derives from the unique continuation principle that

$$
v_{0}=0 \text { in } \mathbb{R}^{d} .
$$

Letting $R \rightarrow \infty$, considering the imaginary part in (2.6), and using (2.3), we obtain

$$
\left\|\nabla v_{\delta}\right\|_{L^{2}\left(\Omega_{2} \backslash \Omega_{1}\right)} \rightarrow 0 \text { as } \delta \rightarrow 0 .
$$

Considering the real part of $(2.6)$ with $R=R_{0}$, we derive from (2.8) and $(2.9)$ that

$$
\left\|v_{\delta}\right\|_{H^{1}\left(B_{R_{0}}\right)} \rightarrow 0 \text { as } \delta \rightarrow 0 .
$$

We have a contradiction by $(2.3)$. Hence $(2.2)$ holds for $R=R_{0}$. The conclusion now follows from 2.5 .

We are ready to give

Proof of Theorem 1.1. Define

$$
u_{1, \delta}=u_{\delta} \circ F^{-1} \text { in } \mathbb{R}^{d} \backslash \Omega_{2},
$$

and

$$
u_{2, \delta}=u_{1, \delta} \circ G^{-1} \text { in } \Omega_{3} .
$$

It follows from (1.13) and a change of variables (see e.g., [16, Lemma 2]) that

$$
\operatorname{div}\left(A \nabla u_{1, \delta}\right)+k^{2} \Sigma u_{1, \delta}+i \delta \operatorname{div}\left(A \nabla u_{1, \delta}\right)=\operatorname{div}\left(A \nabla u_{2, \delta}\right)+k^{2} \Sigma u_{2, \delta}=0 \text { in } \Omega_{3} \backslash \Omega_{2} .
$$

Set

$$
\hat{u}_{\delta}=\left\{\begin{array}{cl}
u_{\delta} & \text { in } \mathbb{R}^{d} \backslash \Omega_{3}, \\
u_{\delta}-\left(u_{1, \delta}-u_{2, \delta}\right) & \text { in } \Omega_{3} \backslash \Omega_{2}, \\
u_{2, \delta} & \text { in } \Omega_{2} .
\end{array}\right.
$$

It follows from 2.10 that $\hat{u}_{\delta} \in H_{l o c}^{1}\left(\mathbb{R}^{d}\right)$ is the unique outgoing solution of

$$
\operatorname{div}\left(\hat{A} \nabla \hat{u}_{\delta}\right)+k^{2} \hat{\Sigma} \hat{u}_{\delta}=f+\operatorname{div}\left(\left[s_{\delta}-s_{0}\right] A \nabla u_{1, \delta}\right) \text { in } \mathbb{R}^{d} .
$$

Since, by Lemma 2.1 .

$$
\left\|u_{\delta}\right\|_{H^{1}\left(B_{R}\right)} \leq C_{R} \delta^{-1}\|f\|_{L^{2}} .
$$

It follows from 2.12 and Lemma 2.1 that

$$
\left\|\hat{u}_{\delta}\right\|_{H^{1}\left(B_{R}\right)} \leq C_{R}\|f\|_{L^{2}} .
$$


As a consequence, we have

$$
\left\|u_{\delta}\right\|_{H^{1}\left(B_{R} \backslash \Omega_{3}\right)} \leq C_{R}\|f\|_{L^{2}} .
$$

Applying [2, Theorem 5.3] (a consequence of a three spheres inequality) for $u_{\delta}$ in $B_{R} \backslash \Omega_{2}$ for some $R>0$ such that $\Omega_{3} \subset \subset B_{R}$, we derive from (2.13) and (2.14) that

$$
\lim _{\delta \rightarrow 0} \delta\left\|u_{\delta}\right\|_{L^{2}\left(B_{R}\right)}=0 .
$$

Using 2.2 in Lemma 2.1, we derive from 2.15) that

$$
\lim _{\delta \rightarrow 0} \delta\left\|u_{\delta}\right\|_{H^{1}\left(B_{R}\right)}=0 ;
$$

which implies

$$
\lim _{\delta \rightarrow 0}\left\|\operatorname{div}\left(\left[s_{\delta}-s_{0}\right] A \nabla u_{1, \delta}\right)\right\|_{H^{-1}}=0 .
$$

A combination of (2.12) and 2.16) yields, for $R>0$,

$$
\left\|\hat{u}_{\delta}-\hat{u}\right\|_{H^{1}\left(B_{R}\right)} \rightarrow 0 \text { as } \delta \rightarrow 0 .
$$

The proof is complete.

Remark 2.1. The same proof gives the same conclusion even if one replaces $f$ by $f_{\delta}$ in $(1.4)$ and assumes that $\left(f_{\delta}\right)$ converges to $f$ in $L^{2}\left(\mathbb{R}^{d}\right)$ and $\operatorname{supp} f_{\delta} \subset \subset B_{R_{0}} \backslash \Omega_{2}$ for some $R_{0}>0$.

Remark 2.2. One of the key points in the proof is the definition of $\hat{u}_{\delta}$ in (2.11) after introducing $u_{1, \delta}$ and $u_{2, \delta}$ as in [19]. In $\Omega_{3} \backslash \Omega_{2}$, we remove $u_{1, \delta}-u_{2, \delta}$ from $u_{\delta}$. The removing term is the singular part of $u_{\delta}$ in $\Omega_{3} \backslash \Omega_{2}$. The way of defining the removing term is intrinsic and more robust than the one in [19], which is based on the separation of variables for a general shell developed there. As seen from there, the removing term becomes more and more singular when one approaches $\partial \Omega_{2}$. The idea of removing the singular term was inspired from the study of the Ginzburg-Landau equation in the work of Bethuel, Brezis, and Helein in [6]. Another new important point in the proof is to establish 2.15). This is obtained by first proving that $u_{\delta}$ is bounded outside $\Omega_{3}$ (again based on the behaviour of $\hat{u}_{\delta}$ ) and then applying a three spheres inequality.

\section{Proof of Proposition 1.1}

We prove Proposition 1.1 by contradiction. Assume that

$$
\limsup _{\delta \rightarrow 0} \delta\left\|\nabla u_{\delta}\right\|_{L^{2}\left(\Omega_{2} \backslash \Omega_{1}\right)}^{2}<+\infty .
$$

Since $\operatorname{div}\left(A \nabla u_{\delta}\right)+k^{2} s_{0} s_{\delta}^{-1} \Sigma u_{\delta}=0$ in $\Omega_{2} \backslash \Omega_{1}$, it follows from a compactness argument that

$$
\left\|u_{\delta}\right\|_{L^{2}\left(\Omega_{2} \backslash \Omega_{1}\right)} \leq C\left\|\nabla u_{\delta}\right\|_{L^{2}\left(\Omega_{2} \backslash \Omega_{1}\right)} .
$$

We derive that

$$
\lim _{\delta \rightarrow 0} \delta\left\|u_{\delta}\right\|_{H^{1}\left(\Omega_{2} \backslash \Omega_{1}\right)}^{2}<+\infty ;
$$


this implies, for $R>0$,

$$
\lim _{\delta \rightarrow 0} \delta\left\|u_{\delta}\right\|_{H^{1}\left(B_{R}\right)}^{2}=0 .
$$

Define

$$
u_{1, \delta}=u_{\delta} \circ F^{-1} \text { in } \Omega_{3} \backslash \Omega_{2}
$$

and set

$$
v_{\delta}=u_{1, \delta}-u_{\delta} \text { in } D .
$$

Then

$$
\begin{gathered}
\operatorname{div}\left(A \nabla v_{\delta}\right)+k^{2} \Sigma v_{\delta}=g_{\delta} \text { in } D, \\
v_{\delta}=0 \text { on } D \cap \partial \Omega_{2} \quad \text { and } \quad A \nabla v_{\delta} \cdot \nu=h_{\delta} \text { on } \partial D \cap \partial \Omega .
\end{gathered}
$$

Here

$$
g_{\delta}=-i \delta \operatorname{div}\left(A \nabla u_{1, \delta}\right)=\frac{i \delta}{1+i \delta} k^{2} \Sigma u_{1, \delta} \text { in } D
$$

and

$$
h_{\delta}=i \delta \nabla u_{1, \delta} \cdot \nu \text { on } \partial D \cap \partial \Omega_{2} .
$$

It is clear from $(3.1)$ that

$$
\delta^{1 / 2}\left\|g_{\delta}\right\|_{L^{2}(D)}+\delta^{1 / 2}\left\|h_{\delta}\right\|_{H^{-1 / 2}\left(\partial D \cap \partial \Omega_{2}\right)} \leq C,
$$

for some $C$ independent of $\delta$. Using (3.3), (3.4), and (3.5), and applying [21, Lemma 10], we have

$$
\limsup _{\delta \rightarrow 0} \delta^{1 / 2}\left\|v_{\delta}\right\|_{H^{1}(D)}=+\infty:
$$

which contradicts (3.2). The proof is complete.

\section{Proof of Proposition 1.2}

We in fact prove a slightly more general result.

Proposition 4.1. Let $d=2,3, \delta \in(0,1), f \in L_{\mathrm{c}}^{2}\left(\mathbb{R}^{d}\right), g \in H^{1 / 2}\left(\partial \Omega_{3}\right)$, and $h \in H^{-1 / 2}\left(\partial \Omega_{3}\right)$. Assume that $\left(s_{0} A, s_{0} \Sigma\right)$ is doubly complementary and $\operatorname{supp} f \cap \Omega_{3}=\varnothing$, and let $V_{\delta} \in H_{\text {loc }}^{1}\left(\mathbb{R}^{d} \backslash\right.$ $\left.\partial \Omega_{3}\right)$ be the unique outgoing solution of

$$
\left\{\begin{array}{l}
\operatorname{div}\left(s_{\delta} A \nabla V_{\delta}\right)+k^{2} s_{0} \Sigma V_{\delta}=f \text { in } \mathbb{R}^{d} \backslash \partial \Omega_{3}, \\
{\left[V_{\delta}\right]=g \quad \text { and } \quad\left[A \nabla V_{\delta} \cdot \eta\right]=h \text { on } \partial \Omega_{3} .}
\end{array}\right.
$$

Then, for $R>0$,

$$
\left\|V_{\delta}\right\|_{H^{1}\left(B_{R} \backslash \partial \Omega_{3}\right)} \leq C_{R}\left(\|f\|_{L^{2}(\Omega)}+\|g\|_{H^{1 / 2}\left(\partial \Omega_{3}\right)}+\|h\|_{H^{-1 / 2}\left(\partial \Omega_{3}\right)}\right),
$$

for some positive constant $C_{R}$ independent of $\delta, f, g$, and $h$. 
Here and in what follows in this paper, we denote $[v]=\left.v\right|_{\text {ext }}-\left.v\right|_{\text {int }}$ and $[M \nabla v \cdot \eta]=$ $\left.M \nabla v \cdot \nu\right|_{\text {ext }}-\left.M \nabla v \cdot \nu\right|_{\text {ext }}$ on $\partial \Omega$ for a smooth bounded open subset $\Omega$ of $\mathbb{R}^{d}$, a matrix-valued function $M$, and for an appropriate function $v$.

It is clear that Proposiiton 4.1 implies Proposition 1.2. Proposition 4.1 is also used in the proof of Proposition 1.3 .

Proof. The proof of Proposition 4.1 has roots from [16] and the key point is to construct a solution $V_{0}$ of 4.1 for $\delta=0$. Let $V \in H_{\text {loc }}^{1}\left(\mathbb{R}^{d} \backslash \partial \Omega_{3}\right)$ be the unique outgoing solution to

$$
\left\{\begin{array}{c}
\operatorname{div}(\hat{A} \nabla \hat{V})+k^{2} \hat{\Sigma} \hat{V}=f \text { in } \mathbb{R}^{d} \backslash \partial \Omega_{3}, \\
{[\hat{V}]=g \quad \text { and } \quad[\hat{A} \nabla \hat{V} \cdot \nu]=h \text { on } \partial \Omega_{3},}
\end{array}\right.
$$

where $(\hat{A}, \hat{\Sigma})$ is defined in 1.16$)$. Then

$$
\|\hat{V}\|_{H^{1}\left(B_{R} \backslash \partial \Omega_{3}\right)} \leq C_{R}\left(\|f\|_{L^{2}}+\|g\|_{H^{1 / 2}\left(\partial \Omega_{3}\right)}+\|h\|_{H^{-1 / 2}\left(\partial \Omega_{3}\right)}\right) .
$$

Define $V_{0} \in H^{1}\left(\Omega \backslash \partial B_{r_{3}}\right)$ as follows

$$
V_{0}=\left\{\begin{array}{cl}
\hat{V} & \text { in } \mathbb{R}^{d} \backslash \Omega_{2}, \\
\hat{V} \circ F & \text { in } \Omega_{2} \backslash \Omega_{1}, \\
\hat{V} \circ G \circ F & \text { in } \Omega_{1} .
\end{array}\right.
$$

Using (1.13) and applying [16, Lemma 2] (a change of variables), as in [16, Step 2 in Section 3.2.2], one can verify that $V_{0} \in H_{\text {loc }}^{1}\left(\mathbb{R}^{d} \backslash \partial \Omega_{3}\right)$ is an outgoing solution to

$$
\operatorname{div}\left(s_{0} A \nabla V_{0}\right)+k^{2} s_{0} \Sigma V_{0}=f \text { in } \mathbb{R}^{d} \backslash\left(\partial \Omega_{3} \cup \partial \Omega_{1}\right) .
$$

One also obtains from the definition of $V_{0}$ and $\hat{V}$ that

$$
\left[V_{0}\right]=g \quad \text { and } \quad\left[A \nabla V_{0} \cdot \nu\right]=h \text { on } \partial \Omega_{3} .
$$

and

$$
\left[V_{0}\right]=0 \quad \text { and } \quad\left[A \nabla V_{0} \cdot \nu\right]=0 \text { on } \partial \Omega_{1} .
$$

Hence $V_{0} \in H_{\text {loc }}^{1}\left(\mathbb{R}^{d}\right)$ is an outgoing solution of 4.1 with $\delta=0$. Set

$$
W_{\delta}=V_{\delta}-V_{0} \text { in } \Omega .
$$

Then $W_{\delta} \in H_{0}^{1}(\Omega)$ is the unique solution to

$$
\operatorname{div}\left(s_{\delta} A \nabla W_{\delta}\right)+k^{2} s_{0} \Sigma W_{\delta}=-\operatorname{div}\left(i \delta A \nabla V_{0} 1_{B_{r_{2}} \backslash B_{r_{1}}}\right) \text { in } \mathbb{R}^{d} .
$$

Here and in what follows, for a subset $D$ of $\mathbb{R}^{d}, 1_{D}$ denotes the characteristic function of $D$. Applying Lemma 2.1, we have

$$
\left\|W_{\delta}\right\|_{H^{1}\left(B_{R}\right)} \leq C_{R}\left\|V_{0}\right\|_{H^{1}\left(\Omega_{2} \backslash \Omega_{1}\right)} .
$$

The conclusion follows from (4.2), 4.3), 4.4), and 4.5). 


\section{$5 \quad$ Proof of Proposition 1.3}

Step 1: Proof of the first statement. Without loss of generality, one might assume that $r_{2}=1$. Define

$$
u_{1, \delta}=u_{\delta} \circ F^{-1} \text { in } \mathbb{R}^{d} \backslash B_{r_{3}},
$$

and

$$
u_{2, \delta}=u_{1, \delta} \circ G^{-1} \text { in } B_{r_{3}} .
$$

Let $\phi \in H^{1}\left(B_{r_{3}} \backslash B_{r_{2}}\right)$ be the unique solution to

$$
\Delta \phi+k^{2} \phi=f \text { in } B_{r_{3}} \backslash B_{r_{2}}, \quad \phi=0 \text { on } \partial B_{r_{2}}, \quad \text { and } \quad \partial_{r} \phi-i k \phi=0 \text { on } \partial B_{r_{3}},
$$

and set

$$
W=w-\phi \text { in } B_{r_{0}} \backslash B_{r_{2}} .
$$

Then $W \in H^{1}\left(B_{r_{3}} \backslash B_{r_{2}}\right)$ satisfies

$$
\Delta W+k^{2} W=0 \text { in } B_{r_{0}} \backslash B_{r_{2}}, \quad W=0 \text { on } \partial B_{r_{2}}, \quad \text { and } \quad \partial_{r} W=-\partial_{r} \phi \text { on } \partial B_{r_{2}} .
$$

We now consider the case $d=2$ and $d=3$ separately.

Case 1: $d=2$. As in [17], define

$$
\hat{J}_{n}(r)=2^{n} n ! J_{n}(r) \quad \text { and } \quad \hat{Y}_{n}(r)=\frac{\pi i}{2^{n}(n-1) !} Y_{n}(r),
$$

where $J_{n}$ and $Y_{n}$ are the Bessel and Neumann functions of order $n$. It follows from [8, (3.57) and (3.58)] that

$$
\hat{J}_{n}(t)=t^{n}[1+o(1)]
$$

and

$$
\hat{Y}_{n}(t)=t^{-n}[1+o(1)]
$$

as $n \rightarrow+\infty$. Since $\Delta W+k^{2} W=0$ in $B_{r_{3}} \backslash B_{r_{2}}$, one can represent $W$ as follows

$$
W=\sum_{n=0}^{\infty} \sum_{ \pm}\left[a_{n, \pm} \hat{J}_{n}(|x|)+b_{n, \pm} \hat{Y}_{n}(|x|)\right] e^{ \pm i n \theta} \quad \text { in } B_{r_{3}} \backslash B_{r_{2}},
$$

for $a_{n, \pm}, b_{n, \pm} \in \mathbb{C}(n \geq 0)$ with $a_{0,+}=a_{0,-}$ and $b_{0,+}=b_{0,-}$. Since $r_{2}=1<r_{0}$ and $W=0$ on $\partial B_{r_{2}}$, we derive from (5.4) and (5.5) that, for some $N>0$ independent of $W$,

$$
\|W\|_{H^{1}\left(B_{r_{0}} \backslash B_{r_{2}}\right)}^{2} \sim \sum_{n=0}^{N} \sum_{ \pm}\left(\left|a_{n, \pm}\right|^{2}+\left|b_{n, \pm}\right|^{2}\right)+\sum_{n=N+1}^{\infty} \sum_{ \pm} n\left|a_{n, \pm}^{2}\right| r_{0}^{2 n}<+\infty .
$$

Here we used the fact $W=0$ on $\partial B_{r_{2}}$ to derive that $a_{n, \pm} \sim b_{n, \pm}$ for $n \geq N+1$ since $r_{2}=1$. One of the keys in the proof is the construction of $W_{\delta} \in H^{1}\left(B_{r_{3}} \backslash B_{r_{2}}\right)$ which is defined as follows

$$
W_{\delta}=\sum_{n=0}^{\infty} \sum_{ \pm} \frac{1}{1+\xi_{n}}\left[a_{n, \pm} \hat{J}_{n}(|x|)+b_{n, \pm} \hat{Y}_{n}(|x|)\right] e^{ \pm i n \theta} \text { in } B_{r_{3}} \backslash B_{r_{2}}
$$


where

$$
\xi_{n}=\delta^{1 / 2}\left(r_{3} / r_{0}\right)^{n} \text { for } n \geq 0 .
$$

Roughly speaking, $W_{\delta}$ is the singularity of $u_{\delta}$. From the definition of $W_{\delta}$, we have

$$
\Delta W_{\delta}+k^{2} W_{\delta}=0 \text { in } B_{r_{3}} \backslash \bar{B}_{r_{2}}, \quad W_{\delta}=0 \text { on } \partial B_{r_{2}},
$$

and

$$
\left\|W_{\delta}\right\|_{H^{1}\left(B_{r_{3}} \backslash B_{r_{2}}\right)}^{2} \sim \sum_{n=0}^{N} \sum_{ \pm}\left(\left|a_{n, \pm}\right|^{2}+\left|b_{n, \pm}\right|^{2}\right)+\sum_{n=N+1}^{\infty} \sum_{ \pm} \frac{n\left|a_{n, \pm}\right|^{2}}{1+\xi_{n}^{2}} r_{3}^{2 n} .
$$

By $(5.9)$, we have, if $\xi_{n} \leq 1$, then

$$
\frac{n\left|a_{n, \pm}\right|^{2}}{1+\xi_{n}^{2}} r_{3}^{2 n} \leq n\left|a_{n, \pm}\right|^{2} r_{3}^{2 n} \leq \delta^{-1} n\left|a_{n, \pm}\right|^{2} r_{0}^{2 n}
$$

and if $\xi_{n} \geq 1$, then

$$
\frac{n\left|a_{n, \pm}\right|^{2}}{1+\xi_{n}^{2}} r_{3}^{2 n} \leq n\left|a_{n, \pm}\right|^{2} r_{3}^{2 n} \xi_{n}^{-2}=\delta^{-1} n\left|a_{n, \pm}\right|^{2} r_{0}^{2 n}
$$

A combination of (5.7), 5.11), 5.12), and (5.13) yields

$$
\left\|W_{\delta}\right\|_{H^{1}\left(B_{r_{3}} \backslash B_{r_{2}}\right)} \leq C \delta^{-1 / 2}
$$

Let $W_{1, \delta} \in H_{l o c}^{1}\left(\mathbb{R}^{2}\right)$ be the unique outgoing solution to

$$
\left\{\begin{array}{c}
\operatorname{div}\left(s_{\delta} A \nabla W_{1, \delta}\right)+k^{2} s_{0} \Sigma W_{1, \delta}=0 \text { in } \mathbb{R}^{2} \backslash \partial B_{r_{2}} \\
{\left[s_{\delta} A \nabla W_{1, \delta} \cdot \nu\right]=(-1-i \delta) h_{\delta} \text { on } \partial B_{r_{2}}}
\end{array}\right.
$$

where

$$
h_{\delta}=-\partial_{r}\left(\phi+W_{\delta}\right) \text { on } \partial B_{r_{2}},
$$

and let $W_{2, \delta} \in H_{\text {loc }}^{1}\left(\mathbb{R}^{2} \backslash \partial B_{r_{3}}\right)$ be the unique outgoing solution to

$$
\left\{\begin{array}{c}
\operatorname{div}\left(s_{\delta} A \nabla W_{2, \delta}\right)+k^{2} s_{0} \Sigma W_{2, \delta}=f 1_{\mathbb{R}^{2} \backslash B_{r_{3}}} \text { in } \mathbb{R}^{2} \backslash \partial B_{r_{3}} \\
{\left[W_{2, \delta}\right]=\phi+W_{\delta} \quad \text { and } \quad\left[A \nabla W_{2, \delta} \cdot \nu\right]=\partial_{r} \phi+\partial_{r} W_{\delta} \text { on } \partial B_{r_{3}} .}
\end{array}\right.
$$

Recall that, for a subset $D$ of $\mathbb{R}^{d}, 1_{D}$ denotes the characteristic function of $D$. From (5.1), (5.10), and the fact $(A, \Sigma)=(I, 1)$ in $B_{r_{3}} \backslash B_{r_{2}}$, we have

$$
u_{\delta}-\left(\phi+W_{\delta}\right) \mathbf{1}_{B_{r_{3}} \backslash B_{r_{2}}}=W_{1, \delta}+W_{2, \delta} \text { in } \Omega .
$$

Using (5.2) and (5.8), we obtain, on $\partial B_{r_{2}}$,

$$
h_{\delta}=-\partial_{r}\left(\phi+W_{\delta}\right)=\partial_{r}\left(W-W_{\delta}\right)=\partial_{r}\left(\sum_{n=0}^{\infty} \sum_{ \pm} \frac{\xi_{n}}{1+\xi_{n}}\left[a_{n, \pm} \hat{J}_{n}(|x|)+b_{n, \pm} \hat{Y}_{n}(|x|)\right] e^{ \pm i n \theta}\right) .
$$


Since $r_{2}=1$, it follows that

$$
\left\|h_{\delta}\right\|_{H^{-1 / 2}\left(\partial B_{r_{2}}\right)}^{2} \lesssim \sum_{n=0}^{N} \sum_{ \pm}\left(\left|a_{n, \pm}\right|^{2}+\left|b_{n, \pm}\right|^{2}\right)+\sum_{n=N+1}^{\infty} \sum_{ \pm} \frac{n\left|\xi_{n}\right|^{2}}{1+\left|\xi_{n}\right|^{2}}\left|a_{n, \pm}\right|^{2} .
$$

Using (5.9), we have, if $\xi_{n} \leq 1$ then

$$
\frac{n\left|\xi_{n}\right|^{2}}{1+\left|\xi_{n}\right|^{2}} \leq \delta n\left|a_{n, \pm}\right|^{2}\left(r_{3} / r_{0}\right)^{2 n}=\delta n r_{0}^{2 n}\left(r_{3} / r_{0}^{2}\right)^{2 n} \leq \delta n r_{0}^{2 n}
$$

since $r_{0}>\sqrt{r_{2} r_{3}}=\sqrt{r_{3}}$, and if $\xi_{n} \geq 1$ then

$$
\frac{n\left|\xi_{n}\right|^{2}}{1+\left|\xi_{n}\right|^{2}} \leq n\left|a_{n, \pm}\right|^{2}=n r_{0}^{2 n} r_{0}^{-2 n} \leq \delta n r_{0}^{2 n}
$$

since $\delta^{1 / 2} r_{0}^{n}>\delta^{1 / 2}\left(r_{3} / r_{0}\right)^{n} \geq 1$. A combination of (5.16), (5.17), and (5.18) yields

$$
\left\|h_{\delta}\right\|_{H^{-1 / 2}\left(\partial B_{r_{2}}\right)} \leq C \delta^{1 / 2}\|W\|_{H^{1 / 2}\left(\partial B_{r_{0}}\right)} \leq C \delta^{1 / 2}
$$

Applying Lemma 2.1, we have

$$
\left\|W_{1, \delta}\right\|_{H^{1}(\Omega)} \leq(C / \delta) \delta^{1 / 2}=C \delta^{-1 / 2} .
$$

On the other hand, from (5.14) and Proposition 4.1, we obtain

$$
\left\|W_{2, \delta}\right\|_{H^{1}\left(B_{r_{3}} \backslash B_{r_{3}}\right)} \leq C \delta^{-1 / 2} .
$$

The conclusion in the case $d=2$ now follows from (5.14), 5.15), (5.19), and 5.20).

Case 2: $d=3$. Define

$$
\hat{j}_{n}(t)=1 \cdot 3 \cdots(2 n+1) j_{n}(t) \quad \text { and } \quad \hat{y}_{n}=-\frac{y_{n}(t)}{1 \cdot 3 \cdots(2 n-1)},
$$

where $j_{n}$ and $y_{n}$ are the spherical Bessel and Neumann functions of order $\mathrm{n}$. Then, as $n$ large enough, (see, e.g., [8, (2.37) and (2.38)])

$$
\hat{j}_{n}(k r)=r^{n}(1+O(1 / n)) \quad \text { and } \quad \hat{y}_{n}(k r)=r^{-n-1}(1+O(1 / n)) .
$$

Thus one can represent $W$ of the form

$$
W=\sum_{n=1}^{\infty} \sum_{-n}^{n}\left[a_{m}^{n} \hat{j}_{n}(|x|)+b_{m}^{n} \hat{y}_{n}(|x|)\right] Y_{m}^{n}(\hat{x}) \quad \text { in } B_{r_{3}} \backslash B_{r_{0}},
$$

for $a_{m}^{n}, b_{m}^{n} \in \mathbb{C}$ and $\hat{x}=x /|x|$. Here $Y_{m}^{n}$ is the spherical harmonic function of degree $n$ and of order $m$. Define $W_{\delta} \in H^{1}\left(B_{r_{3}} \backslash B_{r_{2}}\right)$ by

$$
W_{\delta}=\sum_{n=1}^{\infty} \sum_{-n}^{n} \frac{1}{1+\xi_{n}}\left[a_{m}^{n} \hat{j}_{n}(|x|)+b_{m}^{n} \hat{y}_{n}(|x|)\right] Y_{m}^{n}(\hat{x}) \text { in } B_{r_{3}} \backslash B_{r_{2}},
$$


where

$$
\xi_{n}=\delta^{1 / 2}\left(r_{3} / r_{0}\right)^{n} \text { for } n \geq 1 .
$$

The proof now follows similarly as in the case $d=2$. The details are left to the reader.

Step 2: Proof of the second statement. Define $u_{1, \delta}=u_{\delta} \circ F$ and denote $u_{2^{-n}}$ and $u_{1,2^{-n}}$ by $u_{n}$ and $u_{1, n}$. for notational ease. We prove by contradiction that

$$
\limsup _{n \rightarrow+\infty} 2^{-n / 2}\left(\left\|u_{n}\right\|_{H^{1}\left(B_{r_{3}} \backslash B_{r_{2}}\right)}+\left\|u_{1, n}\right\|_{H^{1}\left(B_{r_{3}} \backslash B_{r_{2}}\right)}\right)=+\infty .
$$

Assume that

$$
m:=\sup _{n} 2^{-n / 2}\left(\left\|u_{n}\right\|_{H^{1}\left(B_{r_{3}} \backslash B_{r_{2}}\right)}+\left\|u_{1, n}\right\|_{H^{1}\left(B_{r_{3}} \backslash B_{r_{2}}\right)}\right)<+\infty .
$$

Define

$$
v_{n}=u_{n}-u_{1, n} \text { in } B_{r_{3}} \backslash B_{r_{2}} \quad \text { and } \quad \phi_{n}=i 2^{-n} \partial_{r} u_{1, n} \text { on } \partial B_{R_{1}} .
$$

Then

$$
\Delta v_{n}+k^{2} v_{n}=f \text { in } B_{r_{3}} \backslash B_{r_{2}}, \quad v_{n}=0 \text { on } \partial B_{r_{2}}, \text { and } \quad \partial_{r} v_{n}=\phi_{n} \text { on } \partial B_{r_{2}} .
$$

We claim that $\left(v_{n}\right)$ is a Cauchy sequence in $H^{1}\left(B_{r_{0}} \backslash B_{r_{2}}\right)$. Indeed, set

$$
V_{n}=v_{n+1}-v_{n} \text { in } B_{r_{3}} \backslash B_{r_{2}} \text { and } \Phi_{n}=\phi_{n+1}-\phi_{n} \text { on } \partial B_{r_{2}} .
$$

We have

$$
\Delta V_{n}+k^{2} V_{n}=0 \text { in } B_{r_{3}} \backslash B_{r_{2}}, \quad V_{n}=0 \text { on } \partial B_{r_{2}}, \quad \text { and } \quad \partial_{r} V_{n}=\Phi_{n} \text { on } \partial B_{r_{2}} .
$$

From (5.25), we derive that

$$
\left\|V_{n}\right\|_{H^{1}\left(B_{R_{2}} \backslash B_{R_{1}}\right)} \leq C m 2^{n / 2} \quad \text { and } \quad\left\|\Phi_{n}\right\|_{H^{1 / 2}\left(\partial B_{R_{1}}\right)} \leq C m 2^{-n / 2} .
$$

In this proof, $C$ denotes a constant independent of $n$. Let $U_{n} \in H^{1}\left(B_{r_{3}}\right)$ be the unique solution of

$$
\Delta U_{n}+k^{2} U_{n}=0 \text { in } B_{r_{3}} \backslash \partial B_{r_{2}}, \quad\left[\partial_{r} U_{n}\right]=\Phi_{n}, \quad \text { and } \quad \partial_{r} U_{n}-i k U_{n}=0 \text { on } \partial B_{r_{3}} .
$$

We have

$$
\left\|U_{n}\right\|_{H^{1}\left(B_{r_{3}}\right)} \leq C\left\|\Phi_{n}\right\|_{H^{-1 / 2}\left(\partial B_{r_{2}}\right)} .
$$

Applying Lemma 5.1 below (a three spheres inequality) for $V_{n} 1_{B_{r_{3}} \backslash B_{r_{2}}}-U_{n}$ in $B_{r_{3}}$, we obtain from 5.26 that

$$
\left\|V_{n}\right\|_{H^{1}\left(B_{r_{0}} \backslash B_{r_{2}}\right)} \leq C\left(\left\|\Phi_{n}\right\|_{H^{-1 / 2}\left(\partial B_{r_{2}}\right)}^{\alpha}\left\|V_{n}\right\|_{H^{1}\left(B_{r_{0}} \backslash B_{r_{2}}\right)}^{1-\alpha}+\left\|\Phi_{n}\right\|_{H^{-1 / 2}\left(\partial B_{r_{2}}\right)}\right) \leq C m 2^{-n \beta},
$$

where $\alpha=\ln \left(r_{3} / r_{0}\right) / \ln \left(r_{3} / r_{1}\right)>1 / 2$ and $\beta=(2 \alpha-1) / 2>0$. Hence $\left(v_{n}\right)$ is a Cauchy sequence in $H^{1}\left(B_{r_{0}} \backslash B_{r_{2}}\right)$. Let $v$ be the limit of $v_{n}$ in $H^{1}\left(B_{r_{0}} \backslash B_{r_{2}}\right)$. Then

$$
\Delta v+k^{2} v=f \text { in } B_{r_{0}} \backslash B_{r_{2}}, \quad v=0 \text { on } \partial B_{R_{1}}, \quad \partial_{r} v=0 \text { on } \partial B_{r} .
$$

This contradicts the non-existence of $v$. Hence (5.24) holds. The proof is complete.

The following lemma is used in the proof of the second statement of Proposition 1.3 . 
Lemma 5.1. Let $d=2,3, k, R>0$, and let $u \in H^{1}\left(B_{R}\right)$ be a solution to the equation $\Delta u+k^{2} u=0$. Then, for $0<R_{1}<R_{2}<R_{3} \leq R$,

$$
\|u\|_{H^{1}\left(B_{R_{2}}\right)} \leq C_{R, k}\|u\|_{H^{1}\left(B_{R_{1}}\right)}^{\alpha}\|u\|_{H^{1}\left(B_{R_{3}}\right)}^{1-\alpha},
$$

where $\alpha=\ln \left(R_{3} / R_{2}\right) / \ln \left(R_{3} / R_{1}\right)$ and $C_{R, k}$ is a positive constant independent of $R_{1}, R_{2}, R_{3}$, and $v$.

Proof. We first give the proof in two dimensions. Since $\Delta v+k^{2} v=0$ in $B_{R}$, one can represent $v$ of the form

$$
v=\sum_{n=0}^{\infty} \sum_{ \pm} a_{n, \pm} \hat{J}_{n}(|x|) e^{ \pm i n \theta} \text { in } B_{R},
$$

for $a_{n, \pm} \in \mathbb{C}(n \geq 0)$ with $a_{0,+}=a_{0,-}$ where $\hat{J}_{n}$ is defined in (5.3). The conclusion now follows from (5.4) after applying Hölder's inequality.

The proof in three dimensions follows similarly. In this case, $v$ can be represented in the form

$$
v=\sum_{n=1}^{\infty} \sum_{-n}^{n} a_{m}^{n} \hat{j}_{n}(|x|) Y_{m}^{n}(\hat{x}) \quad \text { in } B_{R},
$$

for $a_{m}^{n} \in \mathbb{C}$ and $\hat{x}=x /|x|$ where $\hat{j}_{n}$ is defined in (5.21). The conclusion is now a consequence of (5.22) after applying Hölder's inequality.

\section{Cloaking a source via anomalous localized resonance in the finite frequency regime}

In this section, we describe how to use the theory discussed previously to cloak a source $f$ concentrating on an arbitrary bounded smooth manifold of codimension 1 in an arbitrary medium. We follow the strategy in [19]. Without loss of generality, one may assume that the medium is contained in $B_{r_{3}} \backslash B_{r_{2}}$ for some $0<r_{2}<r_{3}$ and characterized by a matrix-valued function $a$ and a real bounded function $\sigma$. We assume in addition that $a$ is Lipschitz and uniformly elliptic in $\overline{B_{r_{3}} \backslash B_{r_{2}}}$ and $\sigma$ is bounded below by a positive constant. Let $f \in L^{2}(\Omega)$ for some bounded smooth open subset $\Omega \subset \subset B_{r_{3}} \backslash B_{r_{2}}$. One might assume as well that $\Omega \subset \subset B\left(x_{0}, r_{0}\right)$ for some $r_{0}>0$ and $x_{0} \in \partial B_{r_{2}}$ where $r_{0}$ is the constant coming from Proposition 1.1. Define $r_{1}=r_{2}^{2} / r_{3}$. Let $F: B_{r_{2}} \backslash\{0\} \rightarrow \mathbb{R}^{d} \backslash B_{r_{2}}$ and $G: \mathbb{R}^{d} \backslash B_{r_{3}} \rightarrow B_{r_{3}} \backslash\{0\}$ be the Kelvin transform with respect to $\partial B_{r_{2}}$ and $\partial B_{r_{3}}$ respectively. Define

$$
A, \Sigma=\left\{\begin{array}{cl}
a, \sigma & \text { in } B_{r_{3}} \backslash B_{r_{2}}, \\
F_{*}^{-1} a, F_{*}^{-1} \sigma & \text { in } B_{r_{2}} \backslash B_{r_{1}}, \\
F_{*}^{-1} G_{*}^{-1} a, F_{*}^{-1} G_{*}^{-1} \sigma & \text { in } B_{r_{1}} \backslash B_{r_{1}^{2} / r_{2}} \\
I, 1 & \text { otherwise. }
\end{array}\right.
$$

It is clear that $\left(s_{0} A, s_{0} \Sigma\right)$ is doubly complementary. Applying Theorem 1.1 and Proposition 1.1, we obtain 
Proposition 6.1. Let $d \geq 2, \delta>0$, and $\Omega \subset \subset D:=B\left(x_{0}, r_{0}\right) \cap\left(B_{r_{3}} \backslash B_{r_{2}}\right)$ be smooth and open, let $f \in L^{2}(\partial \Omega)$ and let $u_{\delta}$ and $v_{\delta}$ be defined by (1.4) and (1.6) where $(A, \Sigma)$ is given in 6.1). Assume that $f \notin \mathcal{H}$ where

$$
\mathcal{H}:=\left\{\left.A \nabla v \cdot \nu\right|_{\partial \Omega} ; v \in H_{0}^{1}(\Omega) \text { is a solution of } \operatorname{div}(A \nabla v)+k^{2} \Sigma v=0 \text { in } \Omega\right\} .
$$

There exists a sequence $\delta_{n} \rightarrow 0$ such that

$$
\lim _{n \rightarrow \infty} E_{\delta_{n}}\left(u_{\delta_{n}}\right)=+\infty
$$

Moreover,

$$
v_{\delta_{n}} \rightarrow 0 \text { weakly in } H_{l o c}^{1}\left(\mathbb{R}^{d} \backslash B_{r_{3}}\right) .
$$

Proof. By Theorem 1.1 and Proposition 1.1, it suffices to prove that there is no $W \in H^{1}(D)$ such that

$$
\operatorname{div}(A \nabla W)+k^{2} \Sigma=f \text { in } D \quad \text { and } \quad W=A \nabla W \cdot \eta=0 \text { on } \partial D \cap \partial B_{r_{2}} .
$$

In fact, Theorem 1.1 and Proposition 1.1 only deal with the case $f \in L^{2}$, however, the same results hold for $f$ stated here and the proofs are unchanged. Suppose that this is not true, i.e., such a $W$ exists. Since $\operatorname{div}(A \nabla W)+k^{2} \Sigma W=0$ in $D \backslash \bar{\Omega}$ and $W=A \nabla W \cdot \nu=0$ on $\partial D \cap \partial B_{r_{2}}$, it follows from the unique continuation principle that $W=0$ in $D \backslash \bar{\Omega}$. Hence

$\left.W\right|_{\Omega} \in H_{0}^{1}(\Omega)$ is a solution of $\operatorname{div}(A \nabla W)+k^{2} \Sigma W=0$ in $\Omega$. We derive that $f=-\left.A \nabla W \cdot \nu\right|_{\Omega}$ on $\partial \Omega$. This contradicts the fact that $f \notin \mathcal{H}$. The proof is complete.

\section{References}

[1] L. Chesnel A. S. Bonnet-Ben Dhia and P. Ciarlet, T-coercivity for scalar interface problems between dielectrics and metamaterials. , ESAIM Math. Model. Numer. Anal. 46 (2012), 1363-1387.

[2] G. Alessandrini, L. Rondi, E. Rosset, and S. Vessella, The stability for the Cauchy problem for elliptic equations, Inverse Problems 25 (2009), 123004.

[3] A. Alu and N. Engheta, Achieving transparency with plasmonic and metamaterial coatings, Phys. Rev. E 95 (2005), 106623.

[4] H. Ammari, G. Ciraolo, H. Kang, H. Lee, and G. W. Milton, Anomalous localized resonance using a folded geometry in three dimensions, Proc. R. Soc. Lond. Ser. A 469 (2013), 20130048.

[5] H. Ammari, G. Ciraolo, H. Kang, H. Lee, and G. W. Milton, Spectral theory of a Neumann-Poincaré-type operator and analysis of cloaking due to anomalous localized resonance, Arch. Rational Mech. Anal. 218 (2013), 667-692.

[6] F. Bethuel, H. Brezis, and F. Helein, Ginzburg-Landau vortices, Progress in Nonlinear Differential Equations and their Applications, vol. 13, Birkhäuser Boston, Inc., Boston, MA, 1994. 
[7] G. Bouchitté and B. Schweizer, Cloaking of small objects by anomalous localized resonance, Quart. J. Mech. Appl. Math. 63 (2010), 437-463.

[8] D. Colton and R. Kress, Inverse acoustic and electromagnetic scattering theory, second ed., Applied Mathematical Sciences, vol. 98, Springer-Verlag, Berlin, 1998.

[9] M. Costabel and S. Ernst, A direct boundary integral equation method for transmission problems, J. Math. Anal. Appl. 106 (1985), 367-413.

[10] H. Kettunen, M. Lassas, and P. Ola, On absence and existence of the anomalous localized resonance without the quasi-static approximation, http://arxiv.org/abs/1406.6224.

[11] R. V. Kohn, J. Lu, B. Schweizer, and M. I. Weinstein, A variational perspective on cloaking by anomalous localized resonance, Comm. Math. Phys. 328 (2014), 1-27.

[12] Y. Lai, H. Chen, Z. Zhang, and C. T. Chan, Complementary media invisibility cloak that cloaks objects at a distance outside the cloaking shell, Phys. Rev. Lett. 102 (2009), 093901.

[13] G. W. Milton, N. A. Nicorovici, R. C. McPhedran, and V. A. Podolskiy, A proof of superlensing in the quasistatic regime, and limitations of superlenses in this regime due to anomalous localized resonance, Proc. R. Soc. Lond. Ser. A 461 (2005), 3999-4034.

[14] G. W. Milton and N. A. Nicorovici, On the cloaking effects associated with anomalous localized resonance, Proc. R. Soc. Lond. Ser. A 462 (2006), 3027-3059.

[15] H-M. Nguyen, Cloaking via change of variables for the Helmholtz equation in the whole spaces, Comm. Pure Appl. Math. 63 (2010), 1505-1524.

[16] H-M. Nguyen, Asymptotic behavior of solutions to the Helmholtz equations with sign changing coefficients, Trans. Amer. Math. Soc. 367 (2015), 6581-6595.

[17] H-M. Nguyen, Superlensing using complementary media, Ann. Inst. H. Poincaré Anal. Non Linéaire 32 (2015), 471-484.

[18] H-M. Nguyen, Cloaking via anomalous localized resonance. A connection between the localized resonance and the blow up of the power for doubly complementary media, C. R. Math. Acad. Sci. Paris 353 (2015), 41-46.

[19] H-M. Nguyen, Cloaking via anomalous localized resonance for doubly complementary media in the quasi static regime, J. Eur. Math. Soc. (JEMS) 17 (2015), 1327-1365.

[20] H-M. Nguyen, Cloaking using complementary media in the quasistatic regime, Ann. Inst. H. Poincaré Anal. Non Linéaire (2015), doi:10.1016/j.anihpc.2015.06.004.

[21] H-M. Nguyen, Limiting absorption principle and well-posedness for the Helmholtz equation with sign changing coefficients, (2015), submitted, arxiv.org/abs/1507.01730.

[22] H-M. Nguyen and H. L. Nguyen, Complete resonance and localized resonance in plasmonic structures, ESAIM: Math. Model. Numer. Anal. 49 (2015), 741-754. 
[23] H-M. Nguyen and H. L. Nguyen, Cloaking using complementary media for the Helmholtz equation and a three spheres inequality for second order elliptic equations, Trans. Amer. Math. Soc. B (2015), to appear, arxiv.org/abs/1504.07378.

[24] N. A. Nicorovici, R. C. McPhedran, and G. W. Milton, Optical and dielectric properties of partially resonant composites, Phys. Rev. B 49 (1994), 8479-8482.

[25] R. A. Shelby, D. R. Smith, and S. Schultz, Experimental Verification of a Negative Index of Refraction, Science 292 (2001), 77-79.

[26] V. G. Veselago, The electrodynamics of substances with simultaneously negative values of $\varepsilon$ and $\mu$, Usp. Fiz. Nauk 92 (1964), 517-526. 